\title{
Effects of lung volume and thoracic gas compression on maximal and partial flow-volume curves
}

\author{
R. Pellegrino*, P. Confessore**, A. Bianco**, V. Brusasco+
}

\begin{abstract}
Effects of lung volume and thoracic gas compression on maximal and partial flow-volume curves. R. Pellegrino, P. Confessore, A. Bianco, V. Brusasco. @ERS Journals Ltd 1996.
\end{abstract}

ABSTRACT: Comparing isovolume flows, measured at the mouth during forced expiratory manoeuvres as started from maximal or partial lung inflation, is a means of assessing the effects of deep inhalation on airway calibre. The aim of this study was to investigate whether the assessment of the effect of deep inhalation during induced bronchoconstriction is influenced by the lung volume at which it is determined and by volume differences due to thoracic gas compression that occur during forced expiratory manoeuvres.

Four healthy subjects and six subjects with mild-to-moderate asthma subjects performed partial and maximal forced expiratory manoeuvres in a flow-type body plethysmograph at control and during a methacholine (MCh) inhalation challenge. Mouth flow $\left(V^{\prime}\right)$ was plotted against both the expired volume $(V \mathrm{mo})$ and the simultaneous thoracic volume measured by plethysmography $\left(V_{\mathrm{pl}}\right)$ changes $\left(V^{\prime}-V_{\text {mo }}\right.$ loop and $V^{\prime}-V \mathrm{pl}$ loop, respectively). The effects of deep inhalation were quantified by determining 1) the ratio of maximal to partial expiratory flows $(\mathrm{M} / \mathrm{P})$ at 30,40 and $50 \%$ of control forced vital capacity (FVC) both on $V^{\prime}-V_{m o}$ loops $\left(\mathrm{M} / \mathbf{P m o}_{\mathrm{m}}\right)$ and $V^{\prime}-V \mathrm{pl}$ loops $(\mathrm{M} / \mathrm{Ppl})$ at control and at MCh end-point; and 2) the slope of the linear regression of maximal vs partial expiratory flows at 30,40 and $50 \%$ of control FVC both on $V^{\prime}-V_{\text {mo loops }}(\mathrm{MPsl}, \mathrm{mo})$ and $V^{\prime}-V_{\mathrm{pl}}$ loops (MPsl,pl) over the entire challenge.

At control, M/Pmo and M/Ppl were similar. At $\mathrm{MCh}$ end-point, $\mathrm{M} / \mathbf{P m o}$ and $\mathrm{M} / \mathrm{Ppl}$ increased more than twofold $(\mathbf{p}<\mathbf{0 . 0 0 2})$, with $\mathrm{M} / \mathrm{Pmo}$ consistently exceeding $\mathrm{M} / \mathrm{Ppl}$ $(\mathrm{p}<0.001)$. In addition, both $\mathrm{M} / \mathrm{Pmo}_{\mathrm{m}}$ and $\mathrm{M} / \mathrm{Ppl}$ varied inversely with lung volume $(\mathrm{p}<0.001)$. By contrast, MPsl,mo and MPsl,pl were not significantly different from each other $(p=0.8)$, and were also similar at the different lung volumes $(p=06)$.

We conclude that during induced bronchoconstriction, the bronchodilation following a deep inhalation, expressed as maximal to partial flow ratio is dependent both on lung volume and volume differences due to thoracic gas compression. The use of expired flow and volume measurements may lead to a small but systematic overestimation of the bronchodilator effect of a deep inhalation. On the contrary, maximal to partial flow slope is insensitive either to lung volume or volume differences due to thoracic gas compression and can, therefore, be fairly determined from expired flow-volume loops.

Eur Respir J., 1996, 9, 2168-2173.

\begin{abstract}
*Servizio di Fisiopatologia Respiratoria, Azienda Ospedaliera S.Croce e Carle, Cuneo, Italy. **Servizio di Fisiopatologia Respiratoria, Reparto di Medicina, Ospedale Militare, Milano, Italy. ${ }^{+}$Cattedra di Fisiopatologia Respiratoria, Dipartimento di Scienze Motorie e Riabilitative, Università di Genova, Genova, Italy.
\end{abstract}

Correspondence: V. Brusasco

Facoltà di Medicina e Chirurgia

Università di Genova

Largo R. Benzi 10

16132 Genova

Italy

Keywords: Asthma

body plethysmography

bronchodilatation

deep inhalation

methacholine

Received: February 271996

Accepted after revision July 41996

Supported in part by a grant from MURST, Rome, Italy.
Measurements of lung mechanics before and after a deep inhalation during induced bronchoconstriction may give substantial information about the ability of the airways to dilate and/or the site of bronchoconstriction [1-7]. Indeed, the changes of airway calibre after deep inhalation depend on the deformable properties of the airways and parenchymal tissue [2], which may be differently affected by spasmogenic stimuli. For example, scarce bronchodilatation after deep inhalation occurs when the bronchoconstriction involves the contractile elements of peripheral airways and/or lung parenchyma, thus increasing parenchymal hysteresis $[2,4]$, or when the forces of interdependence between airways and surrounding parenchyma are weak [7], as well as when airways are inflamed [3].
The method most frequently used to quantify the effect of deep inhalation at a given degree of airway narrowing is the ratio of isovolume flows during expiratory manoeuvres started from maximal or partial lung inflation $(\mathrm{M} / \mathrm{P})$ measured at the mouth $[2-5,8]$. An alternative method is to calculate the slope of the linear regression of the maximal versus the partial flows measured at different levels of airway narrowing (MPsl), which gives information on the changes of the bronchodilator response to deep inhalation as the airway narrowing becomes more severe $[6,9]$. Whether $\mathrm{M} / \mathrm{P}$ and MPsl are dependent on the lung volume at which they are calculated has never been investigated. Were these measurements dependent on lung volume, they would also be dependent on thoracic gas compression, which 
occurs during forced expiratory manoeuvres [10], thus causing a difference between expired volume and thoracic volume changes.

This study was devised to investigate the extent to which lung volume and the volume differences due to thoracic gas compression occurring at control and during induced bronchoconstriction influence the assessment of the effect of deep inhalation on airway calibre when flow-volume curves recorded at the mouth are used.

\section{Methods}

\section{Subjects}

The study was conducted on four healthy volunteers and six mild-to-moderate asthmatic subjects. Their anthropometric and functional characteristics are presented in table 1. All subjects underwent a methacholine (MCh) inhalation challenge. For the asthmatics, this was part of a routine diagnostic procedure. The diagnosis of asthma was made according to the criteria of the American Thoracic Society (ATS) [11]. None of the asthmatics was on steroids or long-acting $\beta_{2}$-agonists at the time of the study. Short-acting $\beta_{2}$-agonists were withheld at least $8 \mathrm{~h}$ before the study [12]. None of the subjects had had an upper respiratory tract infection in the previous 4 weeks. Asthmatics were required to be in a stable clinical condition and to have a forced expiratory volume in one second (FEV1) $>80 \%$ of predicted [13]. All individuals were informed of the purpose of the study and gave their consent.

\section{Methacholine inhalation challenge}

MCh was administered in doubling doses during quiet tidal breathing by using an ampul-dosimeter system (Mefar, Brescia, Italy), which delivers particles with an aerodynamic mass median diameter of $1.53-1.61 \mu \mathrm{m}$, according to the manufacturers. During inspiration, a breath-activated solenoid valve was open for $1 \mathrm{~s}$ and delivered $5 \mu \mathrm{L}$ of solution. The starting doses of $\mathrm{MCh}$ were $0.02 \mathrm{mg}$ for the asthmatic subjects and $0.3 \mathrm{mg}$ for the healthy volunteers. The challenge ended when the FEV1 decreased by $\geq 20 \%$ of control, or upon request by the subject, or when a noncumulative dose of $24 \mathrm{mg}$ was necessary. At the end of the challenge, the subjects were given a $\beta_{2}$-agonist to inhale and dismissed when the FEV1 had returned to within $10 \%$ of control value. No complications occurred during the challenges.

\section{Pulmonary function measurements}

Mouth flow was measured through a heated screentype pneumotachograph (Hans Rudolph Inc., Kansas City, MO, USA) linear up to $12 \mathrm{~L} \cdot \mathrm{s}^{-1}$, connected to a differential pressure transducer (SensorMedics Inc., Yorba Linda, CA, USA), and expired volume was obtained by integration of flow. FEV1 was taken from the best of three acceptable expiratory manoeuvres according to the guidelines of the ATS. Thoracic gas volume at end-tidal expiration was measured in duplicate in a constant volume body plethysmograph (2800 Transmural Body Box; Sensormedics Inc. Yorba Linda, CA, USA) by panting at a frequency $<1 \mathrm{~Hz}$ against a closed shutter. The response of this system is accurate up to $12 \mathrm{~Hz}$. Expiratory reserve volume and inspiratory vital capacity were measured immediately after the opening of the shutter, thus allowing residual volume (RV) and total lung capacity (TLC) to be calculated.

Partial and maximal flow-volume curves were determined with the subjects sitting in the body plethysmograph, which also functions as a flow-type plethysmograph, and mouth flow was measured by the pneumotachograph. Expired lung volume ( $V \mathrm{mo}$ ) was obtained by integrating the mouth flow $\left(V^{\prime}\right)$ signal, while thoracic volume $(V \mathrm{pl})$ changes were obtained from the plethysmograph. The box flow signal was calibrated by using a sinusoidal signal generated by a $50 \mathrm{~mL}$ piston pump at $2 \mathrm{~Hz}$. The mouth flow signal was calibrated by a $3 \mathrm{~L}$ syringe attached to the pneumotachograph. A spirographic tracing was continuously displayed on the screen of a WindoGraph $^{\mathrm{TM}}$ recorder (Gould Inc., Walley View, OH, USA) to provide a visual feedback for the subject and was then printed on paper. All signals were processed by an IBM PS-2 computer and the $V^{\prime}-V_{\mathrm{mo}}$ and $V^{\prime}-V \mathrm{pl}$ loops were superimposed on paper by an Epson EX-1000 graphic printer.

For controls, three repeatable maximal forced expiratory manoeuvres preceded by quiet breathing were obtained at intervals of at least $2 \mathrm{~min}$, in order to calculate inspiratory capacity (IC) and forced vital capacity (FVC). The subjects then performed two sets of three

Table 1. - Anthropometric and pulmonary function data

\begin{tabular}{lcccccccccc}
\hline Subject No. & 1 & 2 & 3 & 4 & 5 & 6 & 7 & 8 & 9 & 10 \\
\hline Status H/A & $\mathrm{H}$ & $\mathrm{A}$ & $\mathrm{H}$ & $\mathrm{A}$ & $\mathrm{H}$ & $\mathrm{H}$ & $\mathrm{A}$ & $\mathrm{A}$ & $\mathrm{A}$ & $\mathrm{A}$ \\
Age yrs & 48 & 42 & 26 & 21 & 28 & 19 & 23 & 19 & 19 & 19 \\
Height cm & 180 & 175 & 180 & 170 & 170 & 185 & 179 & 166 & 168 & 170 \\
Smoke Y/N/EX & $\mathrm{N}$ & $\mathrm{EX}$ & $\mathrm{N}$ & $\mathrm{Y}$ & $\mathrm{N}$ & $\mathrm{N}$ & $\mathrm{Y}$ & $\mathrm{N}$ & $\mathrm{Y}$ & $\mathrm{N}$ \\
FEV L & 4.2 & 4.2 & 4.9 & 3.8 & 3.9 & 5.0 & 4.6 & 3.7 & 3.2 & 3.9 \\
$\quad \%$ pred & 109 & 109 & 111 & 92 & 97 & 102 & 104 & 94 & 81 & 94 \\
FVC L & 5.4 & 6.6 & 5.8 & 5.3 & 4.9 & 5.8 & 5.7 & 4.8 & 3.8 & 5.3 \\
\% pred & 108 & 142 & 112 & 106 & 103 & 101 & 104 & 102 & 81 & 109 \\
TLC L & 7.6 & 8.8 & 7.5 & 6.6 & 7.1 & 7.5 & 8.2 & 6.8 & 5.8 & 7.2 \\
$\quad \%$ pred & 104 & 127 & 105 & 101 & 108 & 100 & 115 & 111 & 92 & 111 \\
PD20 mg & $>24$ & 3.00 & $>5$ & 0.15 & 1.18 & $>2.5$ & 0.14 & 0.05 & 0.04 & 0.13 \\
\hline
\end{tabular}

H/A: healthy/asthmatic; Y/N/EX: smoker/nonsmoker/former smoker; FEV1: forced expiratory volume in one second; FVC: forced vital capacity; TLC: total lung capacity; PD20: noncumulative dose of methacholine that reduced the FEV1 by $20 \%$ of control; \% pred: percentage of predicted volume. 
repeatable manoeuvres a minimum of 2 min apart. The first set consisted of quiet breathing, a forced expiration from about $70 \%$ of FVC to RV, and an inhalation to TLC. The second set consisted of a maximal forced expiratory manoeuvre from TLC to RV, followed by another inhalation to TLC to check for drift. Two minutes after inhaling each dose of MCh, IC was measured in order to calculate the volume between functional residual capacity (FRC) and $70 \%$ of control FVC. Two minutes later, the subjects performed a partial forced expiratory manoeuvre from about $70 \%$ of control FVC to RV and then inhaled to TLC. Two minutes later, the subjects inhaled to TLC and then performed a maximal forced expiration to RV, followed by another inhalation to TLC to check for drift.

\section{Data analysis}

Expiratory flows at 30, 40 and $50 \%$ of control FVC on partial and maximal manoeuvres were measured, assuming that TLC was unchanged over the whole MCh challenge [14]. The ratios of isovolume maximal to partial flows both on $V^{\prime}-V_{\text {mo }}$ loops at 30,40 and $50 \%$ of control FVC (M/P30,mo, M/P40,mo, and M/P5O,mo) and $V^{\prime}-V_{\mathrm{pl}}$, loops (M/P30,pl, M/P40,pl, and M/P50,pl) were then computed at control conditions and at each step of the challenge.

For each individual, maximal flows at 30, 40 and 50\% of control FVC measured over the entire MCh challenge were plotted against the partial flows at the same lung volumes both on $V^{\prime}-V_{\text {mo }}$ and $V^{\prime}-V_{\mathrm{pl}}$, loops, in order to calculate the slope of their linear regression. Thus, the MP30,sl,mo, MP40,sl,mo, and MP50,sl,mo on the $V^{\prime}-V_{\text {mo }}$ loops, and the MP30,sl,pl, MP40,sl,pl, and MP50,sl,pl, on the $V^{\prime}-V \mathrm{pl}$, loops were calculated for each individual. The slopes of such linear regressions are a measure of the bronchodilator effect of deep inhalation over the entire challenge $[6,9]$. For example, if the slope is zero, then for any decrement of partial flow there is no change of maximal flow, indicating that deep inhalation fully reverses the induced bronchoconstriction. If the slope is one, then for any decrement of partial flow there is an equal decrement of maximal flow, indicating that the effect of deep inhalation on airway calibre does not change during the challenge, even when airways narrow.

Airway sensitivity to MCh was inferred from the noncumulative dose of MCh that decreased FEV1 by $20 \%$ of control. This was obtained by linear interpolation between two adjacent points of the dose(log)-response curve.

\section{Statistics}

Data are expressed as mean \pm SD. Comparisons between all measurements were made by two- or three-factor analysis of variance (ANOVA) for repeated measures. The differences between $\mathrm{M} / \mathrm{Pmo}$ and $\mathrm{M} / \mathrm{Ppl}$ and between MPsl,mo and MPsl,pl values were appraised by plotting them against their averages and calculating mean differences \pm 2 SD [15] and linear regression coefficients. Probability values of less than 0.05 were considered statistically significant.

\section{Results}

At MCh end-point, FEV1 and FVC decreased on average by $20 \pm 12$ and $5 \pm 7 \%$ of control, respectively. Maximal forced expiratory flows at 30, 40 and $50 \%$ of the $V^{\prime}-V_{\text {mo }}$ loop decreased by $43 \pm 17,45 \pm 18$ and $44 \pm 23 \%$ of control, respectively. Figure 1 shows typical examples of partial and maximal flow-volume curves measured at the mouth and in the plethysmograph at control and at the end of the challenge in an asthmatic individual.

Both $\mathrm{M} / \mathrm{Pmo}$ and $\mathrm{M} / \mathrm{Ppl}$ values increased with bronchoconstriction at all lung volumes $(\mathrm{p}<0.002)$, with the former being slightly but significantly $(\mathrm{p}<0.001)$ greater than the latter both in asthmatics and normals (table 2). At MCh end-point, both $\mathrm{M} / \mathrm{Pmo}$ and $\mathrm{M} / \mathrm{Ppl}$ were less at high than low volumes in all subjects $(\mathrm{p}<0.001)$. In all but one case, in which it increased, the difference between $\mathrm{M} / \mathrm{Pmo}$ and $\mathrm{M} / \mathrm{Ppl}$ did not change significantly with the average $\mathrm{M} / \mathrm{P}$.

a)

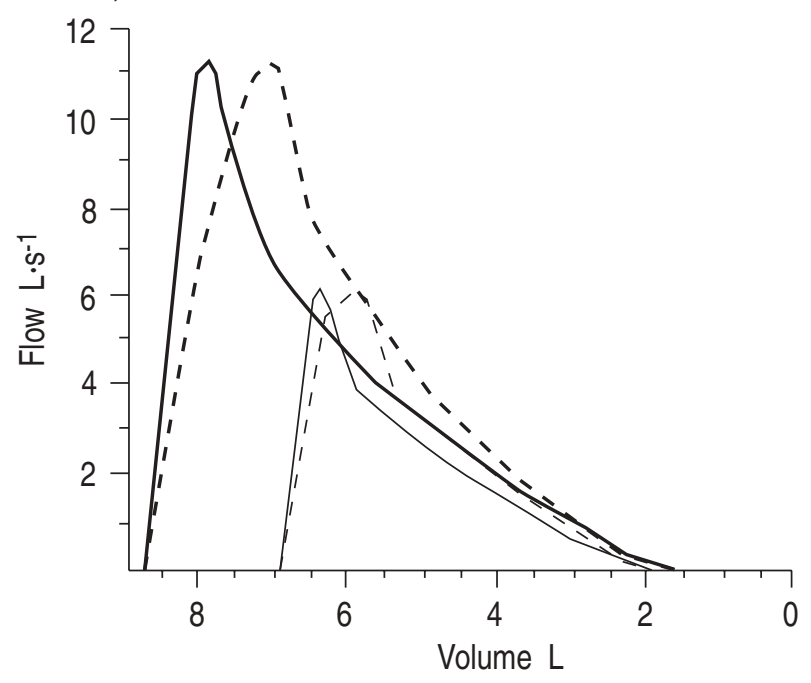

b)

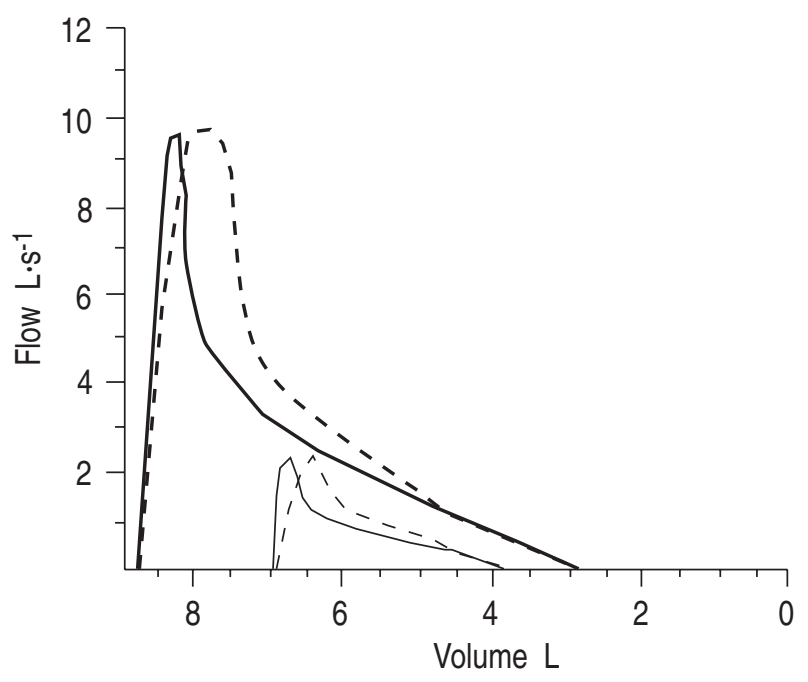

Fig. 1. - Partial (thin lines) and maximal (thick lines) flow-volume curves at the mouth $\left(V^{\prime}-V_{\text {mo; }}\right.$; continuous lines $)$ and by plethysmography ( $V^{\prime}-V_{\mathrm{pl}}$; dashed lines): a) under control conditions; and b) at MCh end-point in an asthmatic. Note that the expired volume $\left(V_{\mathrm{mo}}\right)$ lags behind the decrease in thoracic volume $(V \mathrm{pl})$. 
Table 2. - M/P values at control and at end of the methacholine (MCh) bronchial challenge

\begin{tabular}{lcc}
\hline & Control & MCh end-point \\
\hline M/P30,mo & $1.20 \pm 0.29$ & $4.19 \pm 2.39^{* \#}$ \\
M/P40,mo & $1.21 \pm 0.24$ & $3.28 \pm 1.12^{* \#}$ \\
M/P50,mo & $1.17 \pm 0.24$ & $2.68 \pm 0.49^{* \#}$ \\
M/P30,pl & $1.23 \pm 0.28$ & $3.81 \pm 2.24^{*}$ \\
M/P40,pl & $1.21 \pm 0.27$ & $2.94 \pm 0.95^{*}$ \\
M/P50,pl & $1.18 \pm 0.23$ & $2.39 \pm 0.40^{*}$ \\
\hline
\end{tabular}

M/P30,mo, M/P40,mo, M/P50,mo, M/P30,pl, M/P40,pl, and M/P50,pl: ratios of maximal to partial flows at 30,40 and $50 \%$, respectively, of control forced vital capacity (FVC) calculated on the flow-volume curves at the mouth (mo) and in plethysmography $(\mathrm{pl})$, respectively. Values are presented as mean $\pm \mathrm{SD}$ $(\mathrm{n}=10) . *$ : $\mathrm{p}<0.002$, significantly different from control; \#: $\mathrm{p}<0.001$, significantly different from $\mathrm{M} / \mathrm{Ppl}$ at the same lung volume. At $\mathrm{MCh}$ end-point, there was a significant dependence both of $\mathrm{M} / \mathrm{Pmo}$ and $\mathrm{M} / \mathrm{Ppl}$ values on lung volume $(\mathrm{p}<0.001)$.

a) Healthy
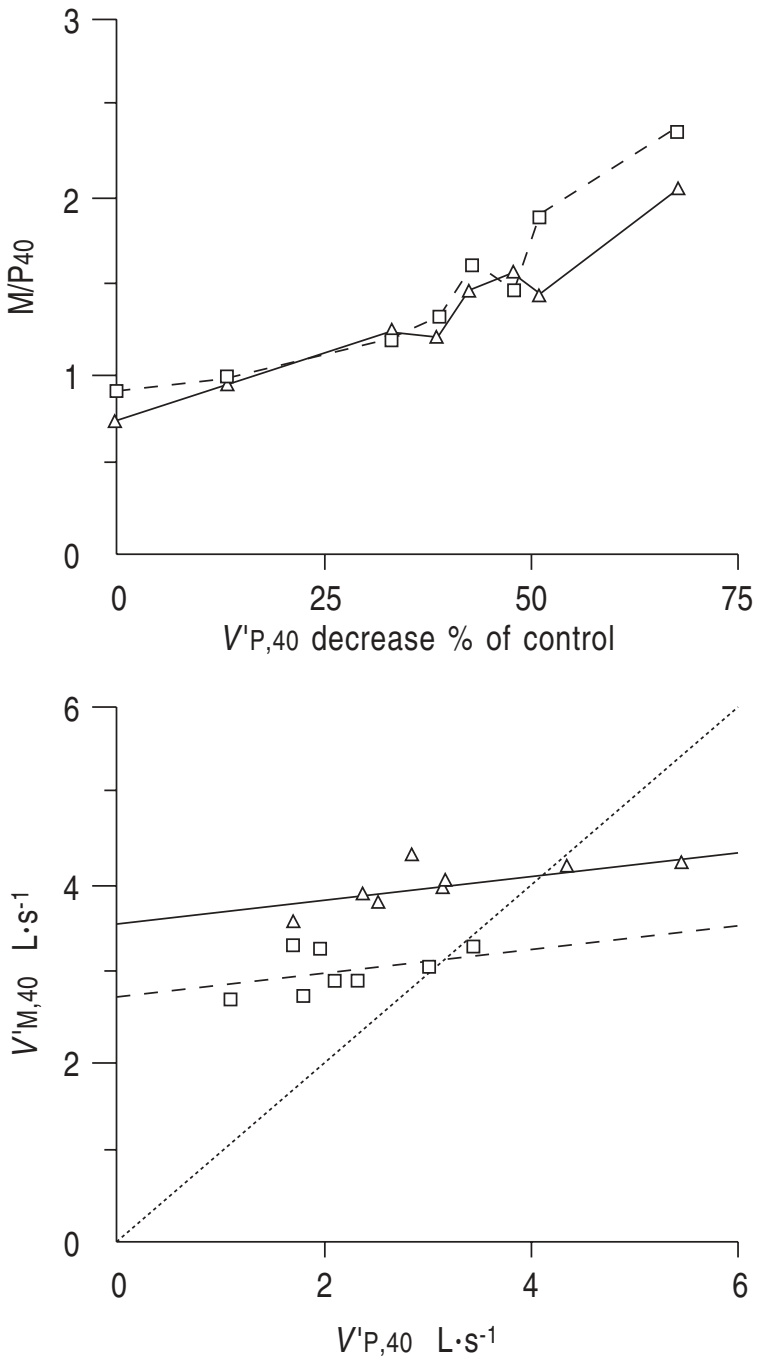

Both MPsl,mo and MPsl,pl values were consistently lower in the healthy than in the asthmatic subjects (fig. 2 ), indicating greater increment of bronchodilatation after deep inhalation as the bronchial tone increased. The MPsl,mo values were similar $(p=0.8)$ to those for MPsl,pl (fig. 3), with no significant effect $(\mathrm{p}=0.6)$ of the lung volume at which they were determined.

\section{Discussion}

The main findings of this study are that during MChinduced bronchoconstriction: 1) M/P increases with decreasing lung volume, and is slightly but consistently greater when calculated from the flow-volumes curves measured at the mouth than by body plethysmography; and 2) MPsl is independent of lung volume, and similar when determined at the mouth or by body plethysmography.

\section{b) Asthmatic}
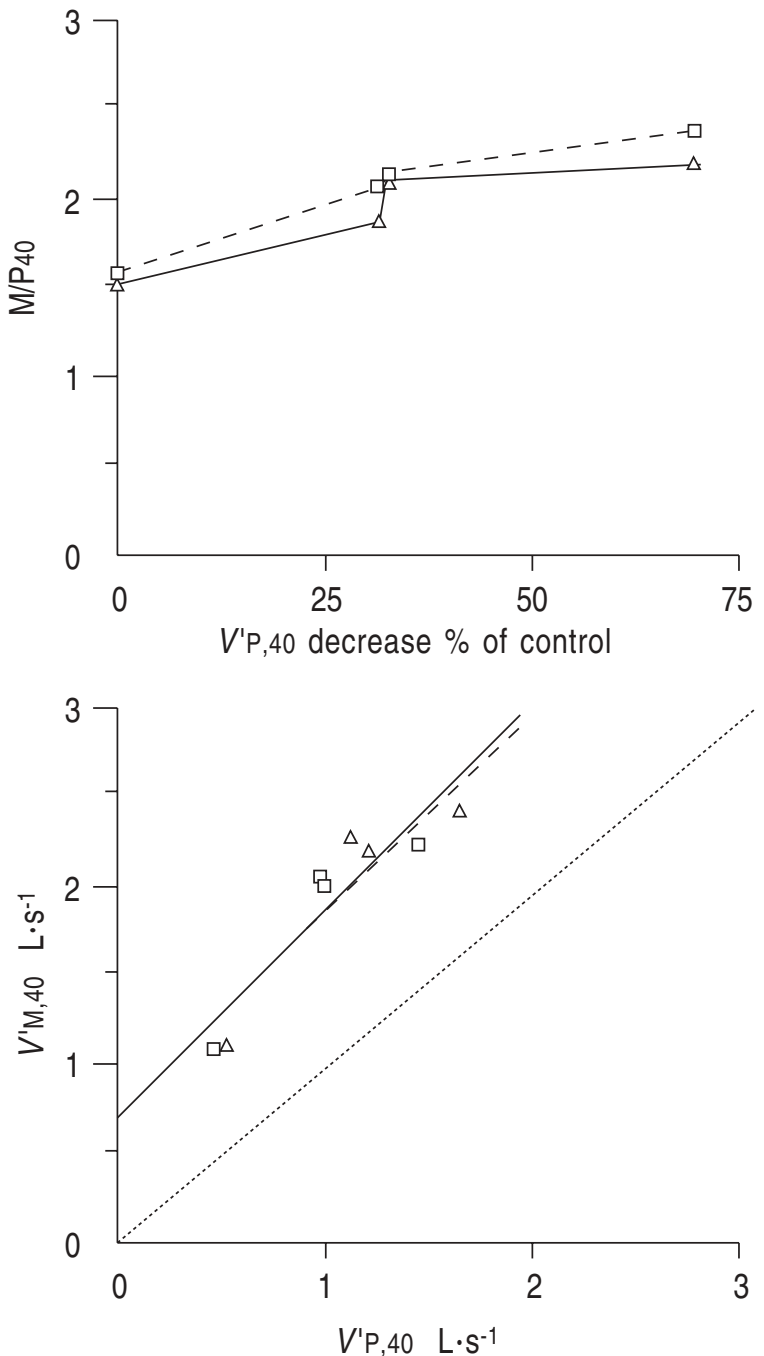

Fig. 2. - Upper panels: ratios of maximal to partial flows at $40 \%$ of control forced vital capacity (M/P40) measured on $V^{\prime}-V_{\text {mo }}(\mathrm{M} / \mathrm{P} 40$,mo; $\square$ ) and on $V^{\prime}-V_{\mathrm{pl}}$ loops $(\mathrm{M} / \mathrm{P} 40, \mathrm{pl} ; \Delta)$ plotted against the percentage decrease of partial expiratory flow measured on $V^{\prime}-V_{\mathrm{mo}}\left(V^{\prime} \mathrm{P}, 40\right)$ during bronchoconstriction in two representative individuals a) healthy; and b) asthmatic. Note that the both $\mathrm{M} / \mathrm{P} 40$,mo and $\mathrm{M} / \mathrm{P} 40$,pl increase with bronchoconstriction, but $\mathrm{M} / \mathrm{P} 40$,mo increases more than $\mathrm{M} / \mathrm{P} 40$,pl. Lower panels: plots of maximal $\left(V^{\prime} \mathrm{M}, 40\right) v s$ partial $\left(V^{\prime} \mathrm{P}, 40\right)$ forced expiratory flows at $40 \%$ of control forced vital capacity measured on $V^{\prime}-V_{\text {mo }}(\square)$ and $V^{\prime}-V_{\mathrm{pl}}$ loops $(\Delta)$ in the same subjects. The continuous and dashed straight lines are the slopes of the linear regressions of the data recorded at the mouth (MP40,sl,mo) and by plethysmography (MP40,sl,pl), respectively. The dotted oblique lines are the lines of identity. Note that MP40,sl,mo and MP40,sl,pl are similar in the individual subjects, but less in the healthy than in the asthmatic subject, indicating a greater progressive bronchodilator effect of deep inhalation in the former. 

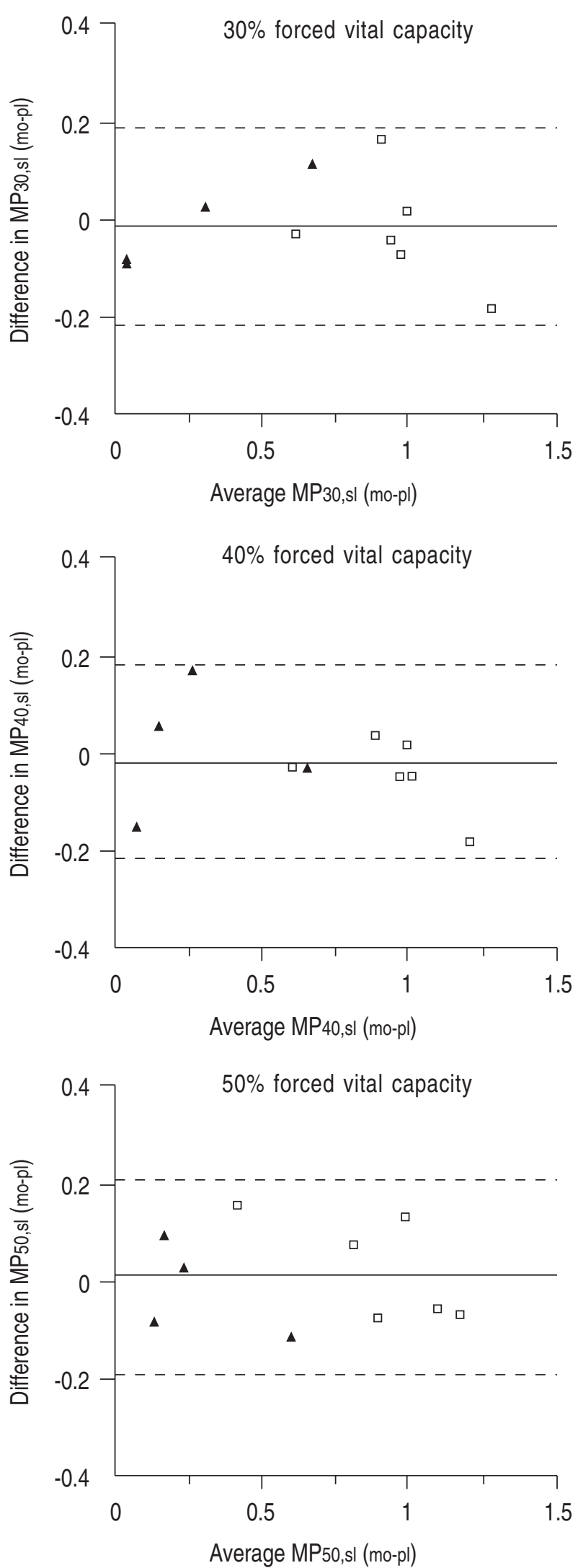

Fig. 3. - Plots of the differences between MPsl,mo and MPsl,pl vs their averages in the normal $(\boldsymbol{\Delta})$ and asthmatic $(\square)$ individuals at different lung volumes $(30,40$ and $50 \%$ of forced vital capacity). Mean differences (-) with $\pm 2 \mathrm{SD}$ (---) are indicated. MPsl,mo and MPsl,pl: maximum to partial slope of linear regression of data recorded at the mouth and by plethysmography, respectively.
The volume of thoracic gas compressed during forced expiration at a given lung volume depends on airway resistance [10]. This predicts that the volume difference due to gas compression increases during bronchoconstriction. Furthermore, as deep inhalation may cause either bronchodilatation or bronchoconstriction [2-6, 9], the volume of thoracic gas compression may differ during maximal and partial forced expiratory manoeuvres. Consistent with this prediction, gas compression was greater during maximal than partial manoeuvres in subjects with chronic airway obstruction and deep inhalation induced bronchoconstriction [8]. The opposite might be expected during induced bronchoconstriction, when deep inhalation has a bronchodilator effect [2].

$\mathrm{M} / \mathrm{P}$ has been widely-used to assess the effect of deep inhalation under different conditions [2-5, 8]. Generally, the lung volume at which $\mathrm{M} / \mathrm{P}$ was determined ranged $30-50 \%$ of FVC. It can be assumed that $\mathrm{M} / \mathrm{P}$ is independent of lung volume only if partial and maximal flow-volume curves have linear downslopes and terminate at the same RV. This may be the case in healthy or mild asthmatic individuals under control conditions, but not during induced bronchoconstriction when deep inhalation increases both expiratory flows and FVC [5]. Under these circumstances, unless the down slopes of partial and maximal manoeuvres are substantially different, $\mathrm{M} / \mathrm{P}$ increases as lung volume decreases. This was indeed the case in the present study. Accordingly, the volume change due to thoracic gas compression after MCh caused M/Pmo values to exceed those of $\mathrm{M} / \mathrm{Ppl}$, the former actually being measured at lower lung volumes. However, a greater thoracic gas compression on partial than maximal manoeuvres would also have resulted in $\mathrm{M} / \mathrm{Pmo}$ values larger than those of $\mathrm{M} / \mathrm{Ppl} \mathrm{s}$.

MPsl is an index which has recently been proposed to quantify the bronchodilator effect of deep inhalation during bronchial challenge $[6,9]$. It has the advantage of taking into account the dilator response at all steps of the challenge, which makes it independent of the level of airway narrowing at which the response is considered. It is assessed from the slope of the linear regression of the maximal and partial expiratory flows [9] or their percentage changes from control [6]. In the present study, MPsl measurements were independent of the lung volume at which they were taken, and similar when measured at the mouth or by plethysmography. The insensitivity of MPsl to lung volume is likely because it is calculated on absolute differences between maximal and partial flows, which are not affected by changes in FVC. The similarity between MPsl,mo and MPsl,pl values suggests that thoracic gas compression was similar on partial and maximal manoeuvres throughout the challenge, as greater volume differences due to thoracic gas compression on partial than maximal manoeuvres with bronchoconstriction would have caused MPsl,mo to exceed MPsl,pl. Indeed, no differences in the amount of thoracic gas compressed was detectable between maximal and partial manoeuvres.

Reliable assessment of the effects of deep inhalation during induced bronchoconstriction may predict the level at which bronchoconstriction is limited, as well as exaggerated airway narrowing [6]. Moreover, the changes of airway calibre after deep inhalation represent a potential and useful means for evaluating the benefits of the 
anti-inflammatory therapy in subjects with chronic bronchoconstriction [16].

The present study shows that measuring maximal to partial flow ratio at the mouth may result in a systematic overestimation of the bronchodilator effect of deep inhalation because of the volume difference due to thoracic gas compression. This error is, however, relatively small (10-12\%) and may not lead to substantially erroneous conclusions. Nevertheless, the bronchodilator effect of deep inhalation during induced bronchoconstriction can be more reliably inferred from maximal to partial flow slope, as this is not sensitive to lung volume or to volume changes due to thoracic gas compression.

Acknowledgement: The authors are grateful to R. Perissin and SensorMedics Italia for technical assistance.

\section{References}

1. Fish JE, Ankin ML, Kelly IF, Peterman VI. Regulation of bronchomotor tone by lung inflation in asthmatics and nonasthmatic subjects. J Appl Physiol: Respirat Environ Exercise Physiol 1981; 50: 1079-1086.

2. Burns CB, Taylor WR, Ingram RH. Effects of deep inhalation in asthma: relative airway and parenchymal hysteresis. J Appl Physiol 1985; 59: 1590-1596.

3. Pliss LB, Ingenito EP, Ingram RH Ir. Responsiveness, inflammation, and effects of deep breath on obstruction in mild asthma. J Appl Physiol 1989; 66: 2298-2304.

4. Brusasco V, Pellegrino R, Violante B, Crimi E. Relationship between quasistatic pulmonary hysteresis and maximal airway narrowing in humans. J Appl Physiol 1992; 72: 2075-2080.

5. Pellegrino R, Violante B, Selleri R, Brusasco V. Changes in residual volume during induced bronchoconstriction in healthy and asthmatic subjects. Am J Respir Crit Care Med 1994; 150: 363-368.

6. Pellegrino R, Violante B, Brusasco V. Maximal bronchoconstriction in humans: relationship to deep inhalation and airway sensitivity. Am J Respir Crit Care Med 1996; 153: $115-121$.

7. Skloot G, Permutt S, Togias AG. Airway hyperresponsiveness in asthma: a problem of limited smooth muscle relaxation with inspiration. J Clin Invest 1995; 96 : 2393-2403.

8. Fairshter RD, Berry RB, Wilson AF, Brideshead T, Mukai D. Effects of thoracic gas compression on maximal and partial flow-volume maneuvers. J Appl Physiol 1989; 67: 780-785.

9. Pellegrino R, Wilson O, Jenouri G, Rodarte JR. Deep inhalation during bronchoconstriction. Am J Respir Crit Care Med 1994; 149, A879.

10. Ingram RH, Schilder DP. Effects of thoracic gas compression on the flow-volume curve of the forced vital capacity. Am Rev Respir Dis 1966; 94: 56-63.

11. American Thoracic Society. Chronic bronchitis, asthma and pulmonary emphysema. Am Rev Respir Dis 1962; 84: 762-768.

12. Eiser NM, Kerrebijn KF, Quanjer PH. Guidelines for standardization of bronchial challenges with (nonspecific) bronchoconstricting agents. Eur Respir J 1983; 19: 495-514.

13. Quanjer PH, Tammelin GJ, Cotes JE, Pedersen OF, Peslin R, Yernault J-C. Lung volumes and forced ventilatory flows. Report Working Party "Standardization of Lung Function Tests". European Coal and Steel Community. Eur Respir J 1993; 6 (Suppl. 16): 5-40.

14. Kirby JB, Juniper EF, Hargreave FE, Zamel N. Total lung capacity does not change during methacholine-stimulated airway narrowing. J Appl Physiol 1986; 61: 2144-2147.

15. Bland JM, Altman DG. Statistical methods for assessing agreement between two methods of clinical measurement. Lancet 1986; 8: 307-310.

16. Bel EH, Timmers MC, Hermans J, Dijkman JH, Sterk PJ. The long-term effects of nedocromil sodium and beclomethasone dipropionate on bronchial responsiveness to methacholine in nonatopic asthmatic subjects. Am Rev Respir Dis 1990; 141: 21-28. 